\author{
Martin W. Dünser \\ Jukka Takala \\ Hanno Ulmer \\ Viktoria D. Mayr \\ Günter Luckner \\ Stefan Jochberger \\ Fritz Daudel \\ Philipp Lepper \\ Walter R. Hasibeder \\ Stephan M. Jakob
}

\title{
Arterial blood pressure during early sepsis and outcome
}

Received: 29 May 2008

Accepted: 19 January 2009

Published online: 3 February 2009

(C) Springer-Verlag 2009

Electronic supplementary material The online version of this article

(doi:10.1007/s00134-009-1427-2) contains supplementary material, which is available to authorized users.

M. W. Dünser ( $)$ J. Takala · F. Daudel · P. Lepper · S. M. Jakob

Department of Intensive Care Medicine, Inselspital, 3010 Bern, Switzerland e-mail: Martin.Duenser@i-med.ac.at

Tel.: +41-31-6322111

Fax: +41-31-63229644

H. Ulmer

Institute of Medical Biostatistics, Innsbruck Medical University, Innsbruck, Austria

V. D. Mayr - G. Luckner - S. Jochberger Department of Anesthesiology and Critical Care Medicine, Innsbruck Medical

University, Innsbruck, Austria

W. R. Hasibeder

Department of Anesthesiology and Critical Care Medicine, Krankenhaus Ried im Innkreis, Ried im Innkreis, Austria
Abstract Objective: To evaluate the association between arterial blood pressure $(\mathrm{ABP})$ during the first $24 \mathrm{~h}$ and mortality in sepsis.

Design: Retrospective cohort study. Setting: Multidisciplinary intensive care unit (ICU). Patients and participants: A total of 274 septic

patients. Interventions: None. Measurements and

results: Hemodynamic, and laboratory parameters were extracted from a PDMS database. The hourly time integral of ABP drops below clinically relevant systolic arterial pressure (SAP), mean arterial pressure (MAP), and mean perfusion pressure $(\mathrm{MPP}=\mathrm{MAP}-$ central venous pressure) levels was calculated for the first $24 \mathrm{~h}$ after ICU admission and compared with 28day-mortality. Binary and linear regression models (adjusted for SAPS II as a measure of disease severity), and a receiver operating characteristic (ROC) analysis were applied. The areas under the ROC curve were largest for the hourly time integrals of
ABP drops below MAP $60 \mathrm{mmHg}$ (0.779 vs. 0.764 for ABP drops below MAP $55 \mathrm{mmHg} ; P \leq 0.01$ ) and MPP $45 \mathrm{mmHg}$. No association between the hourly time integrals of $\mathrm{ABP}$ drops below certain SAP levels and mortality was detected. One or more episodes of MAP $<60 \mathrm{mmHg}$ increased the risk of death by 2.96 (CI $95 \%, 1.06-10.36, P=0.04)$. The area under the ROC curve to predict the need for renal replacement therapy was highest for the hourly time integral of ABP drops below MAP $75 \mathrm{mmHg}$. Conclusions: A MAP level $\geq 60 \mathrm{mmHg}$ may be as safe as higher MAP levels during the first $24 \mathrm{~h}$ of ICU therapy in septic patients. A higher MAP may be required to maintain kidney function.

Keywords Hypotension. Mean arterial blood pressure . Mean perfusion pressure $\cdot$ Sepsis

\section{Introduction}

Cardiovascular failure is a major determinant of outcome [1-3]. Arterial blood pressure (ABP) is widely used to monitor and guide hemodynamic therapy. Although numerous studies have evaluated different strategies to increase $\mathrm{ABP}[4,5]$, few data exist to which level $\mathrm{ABP}$ should be elevated. In septic shock, mean arterial blood pressure (MAP) targets between $65-90 \mathrm{mmHg}$ have been proposed [6-8]. The ABP levels achieved in various studies appear to be remarkably higher, e.g. $80-95 \mathrm{mmHg}$ $[9,10]$, than those specified in the respective protocols. Determination of a clinically relevant ABP target could influence the extent of pharmacological support in 
critically ill sepsis patients. So far, only two small prospective studies including a total of 38 patients evaluated tissue perfusion at different ABPs [11, 12].

A retrospective study by Varpula et al. [13] addressed hemodynamic treatment goals in 111 septic shock patients. The association between the area below threshold values of specific cardiovascular variables during the first $48 \mathrm{~h}$ of septic shock and 30-day-mortality was assessed, without adjustment for disease severity. The area below a MAP of $65 \mathrm{mmHg}$ and a mixed venous oxygen saturation of $70 \%$ was most predictive of 30-daymortality.

Since ABP targets in septic shock remain controversial, we evaluated the association between different ABP levels during the first $24 \mathrm{~h}$ after ICU admission and 28day-mortality and organ function in 274 sepsis patients using a disease severity adjusted model. We hypothesized that lower ABPs than commonly targeted may be welltolerated. Parts of this work were presented as an abstract at the twentieth Congress of the European Society of Intensive Care Medicine [14].

\section{Patients and methods}

This retrospective, explorative cohort study was performed in a 30-bed multi-disciplinary ICU in a university hospital. All medical records from 1 January 2005 until 30 June 2006 were reviewed for patients admitted to the ICU because of sepsis defined according to the American College of Chest Physicians and the Society of Critical Care Medicine [15]. Patients $<18$ years, discharged alive $<24 \mathrm{~h}$ after ICU admission, or developed sepsis during their ICU stay were excluded. The study protocol was approved by the Ethic Committee of the Canton Bern.

All study variables were extracted from the institutional patient data management system database (Centricity Critical Care Clinisoft ${ }^{\circledR}$; Deio, Kuopio, Finland). Routine data recording includes demographic and clinical patient characteristics. Hemodynamic parameters are prospectively recorded. The system uses median filtering which is an effective non-linear, digital filtering process to eliminate artefacts from a signal. Thus, single ABP values over 2 min are summarized as a median value [16]. All laboratory results are automatically imported into the system. Drugs and fluids administered are manually entered into the database at the bedside.

\section{Hemodynamic therapy}

An arterial, a central venous and a pulmonary artery catheter was in place in 274 (100\%), 227 (82.8\%), and 74 (27\%) patients, respectively. Hemodynamic therapy of study patients was based on an institutional protocol which served as a treatment guideline (Electronic Repository). According to this, all patients received repetitive fluid challenges based on the response of $\mathrm{ABP}$, heart rate, central venous pressure, mixed venous oxygen saturation, and the adequacy of peripheral circulation. If signs of tissue hypoperfusion (lactic acidosis, signs of peripheral vasoconstriction) persisted and catecholamines were required, a pulmonary artery catheter (Swan Ganz $\mathrm{CCOmbo}^{\circledR} \mathrm{CCO} / \mathrm{SvO}_{2} / \mathrm{VIP}$; Edwards Lifesciences Inc., Irvine, CA, USA) with continuous cardiac output measurement (Vigilance ${ }^{\circledR}$; Edwards Lifesciences Inc., Irvine, CA, USA) was inserted. In these patients, fluid loading was guided according to changes of stroke volume index and mixed venous oxygen saturation in response to volume infusion (predominantly colloids). If mixed venous oxygen saturation remained $<60 \%$ despite optimal fluid resuscitation and acceptable hemoglobin, inotropic therapy with dobutamine (second line agent, epinephrine) was initiated. If MAP remained $<55-60 \mathrm{mmHg}$ despite the use of volume and inotropes, norepinephrine was installed. Individual MAP targets were set between 50 and $75 \mathrm{mmHg}$ according to urine output, arterial lactate levels, adequacy of peripheral circulation, and mixed venous oxygen saturation.

\section{Study variables}

Demographic data, pre-existent diseases, source of infection and in case of surgery incidence and type of operation were documented. Septic shock was defined as the presence of sepsis together with persistent arterial hypotension which could not be explained by other causes and required the infusion of inotropic and/or vasopressor agents [14]. SAPS II [17] and Acute Physiology and Chronic Health Evaluation (APACHE) II [18] were calculated from worst clinical parameters during the first $24 \mathrm{~h}$ after admission. Length of ICU and hospital stay, and patient outcome at ICU discharge were recorded. Twentyeight day mortality was calculated from ICU records, the hospital database, or in case of transfer to external institutions before day 28 by contacting these hospitals.

\section{Hemodynamic data documentation}

Manual quality and plausibility control of individual datasets was performed to exclude artefacts (e.g. due to blood sampling via the arterial line). We have previously demonstrated that clinicians can efficiently detect artefacts in monitored trends [19]. Mean hemodynamic values during the first $24 \mathrm{~h}$ after ICU admission were calculated. Mean perfusion pressure $(\mathrm{MPP}=\mathrm{MAP}-$ central venous pressure) and in patients with a pulmonary artery catheter, systemic vascular resistance index was calculated. 
For systolic arterial blood pressure (SAP), MAP, and MPP the blood pressure time integral of the measured ABP curve during the first $24 \mathrm{~h}$ was calculated (Electronic Repository). Because of differences in the actual recorded ABP time due to diagnostic and/or operative procedures, the integral was normalized for the time recorded (hourly integral) for each variable was introduced into the statistical model. In case of death within $24 \mathrm{~h}$, hemodynamic variables during the last $30 \mathrm{~min}$ before cardiac arrest and variables recorded after the decision to withdraw life-sustaining therapy were excluded. The hourly time integral of ABP drops below certain threshold levels of SAP $(95,90,85,80,75,70$, $65 \mathrm{mmHg})$, MAP $(75,70,65,60,55,50,45 \mathrm{mmHg})$ and MPP $(60,55,50,45,40,35,30 \mathrm{mmHg})$ was calculated. Wherever possible, individually prescribed MAP targets were recorded (Electronic Repository) and the hourly time integral of ABP drops below this target was calculated. Application of cardiovascular active drugs, analgosedative medications, and diuretics during the observation period was documented in a binary fashion.

\section{Laboratory data documentation}

The most aberrant standard laboratory parameters during the ICU stay were extracted from the database. Hourly urine output during the first $24 \mathrm{~h}$ and need for renal replacement therapy (decisions to start renal replacement therapy for acute renal failure were made together with a nephrologist) were recorded. The Sequential Organ Failure Assessment (SOFA) score [20] was calculated daily from given clinical and laboratory parameters. Organ failure was defined as greater than three points in each organ subscore of SOFA.

\section{Study endpoints}

The primary endpoint was to evaluate the SAP, MAP and MPP levels during the first $24 \mathrm{~h}$ after ICU admission which were associated best with 28-day-mortality. The secondary endpoint was to evaluate the association between ABPs during the first $24 \mathrm{~h}$ after ICU admission and organ function.

\section{Statistical analysis}

All statistical analyses were performed with the SPSS 12.0.1. (SPSS, Chicago, IL, USA) and STATA software 9.2. (StataCorp, College Station, TX, USA). Kolmogorov-Smirnov tests were used to check for normality distribution. In case of non-normal distribution, logarithmic transformation was used. As appropriate, unpaired student's $t$ and $\chi^{2}$ tests were used to compare data between survivors and nonsurvivors and other subgroups, respectively.

A binary logistic regression model was used to evaluate the association between the hourly blood pressure time integral of measured ABPs as well as the hourly time integral of ABP drops below certain ABP levels and 28day-mortality or organ failures. To account for disease severity, the SAPS II (excluding SAP count) was entered into the models as a covariate. The area under the receiver operator characteristic (ROC) curve, sensitivity, specificity, and negative and positive predictive values was determined from the final classification tables of the logistic regression models. The ABP level below which the hourly time integral of ABP drops showed the largest area under the ROC curve was considered to have the best association with 28-day-mortality and was subsequently compared with other ABP levels in the same group (SAP, MAP, MPP) using an algorithm suggested by DeLong et al. [21].

For continuous variables (e.g. maximum laboratory values), a linear regression model including SAPS II (excluding SAP count) as a covariate was applied. Patients with chronic renal insufficiency (elevated baseline creatinine concentration) and patients receiving diuretics during the observation period were excluded from the regression analyses evaluating the association between ABP and creatinine serum concentrations and urine output/h, respectively. Patients on chronic hemodialysis were excluded from the model for the assessment of the association between ABP and need for renal replacement therapy. In order to exclude collinearity, age, the hourly cardiac index time integral, chronic arterial hypertension (documented history and/or repeatedly measured increased values according to WHO limits before the septic episode), need for catecholamines and analgosedative drugs were entered as covariates into single logistic regression models.

For simple comparisons, $P$ values $<0.05$ were considered to indicate statistical significance. In case of multiple comparisons, $P$ values $<0.007$ (regression analyses evaluating the association between seven ABP levels and 28-day-mortality as well as organ function) were applied. Data are given as mean values $\pm \mathrm{SD}$, if not indicated otherwise.

\section{Results}

During the observation period, 4,590 patients were admitted to the ICU. A total of 274 patients fulfilled the inclusion criteria and were included in the analysis (Table 1). SAP and MAP were recorded for $21.6 \pm 3.6 \mathrm{~h}$, MPP for $18.9 \pm 5.2 \mathrm{~h}$ (Electronic Repository). Seven study patients died during the $24 \mathrm{~h}$ observation period. Patients with septic shock had a higher ICU-(25.7 vs. 
Table 1 Characteristics of the study population

\begin{tabular}{|c|c|}
\hline$n$ & 274 \\
\hline Age (years) & $61 \pm 16$ \\
\hline Male sex, $n(\%)$ & $170(62)$ \\
\hline \multicolumn{2}{|l|}{ Premorbidites, $n(\%)$} \\
\hline cAHT & $104(38)$ \\
\hline CHD & $62(22.6)$ \\
\hline $\mathrm{CHF}$ & $41(15)$ \\
\hline COPD & $48(17.5)$ \\
\hline CRI & $37(13.5)$ \\
\hline CLD & $26(9.5)$ \\
\hline Neoplasm & $50(18.2)$ \\
\hline \multicolumn{2}{|c|}{ Origin of ICU admission, $n(\%)$} \\
\hline Ward & $92(33.6)$ \\
\hline Operation room & $82(30)$ \\
\hline Other hospital & $75(27.4)$ \\
\hline Emergency department & $25(9.1)$ \\
\hline \multicolumn{2}{|l|}{ Source of infection, $n(\%)$} \\
\hline Lungs & $111(40.5)$ \\
\hline Urinary tract & $14(5.1)$ \\
\hline Skin/soft tissue & $27(9.9)$ \\
\hline Liver/abdomen & 73 (26.7) \\
\hline Catheter/device & $9(3.3)$ \\
\hline CNS & $15(5.5)$ \\
\hline Endocarditis & $13(4.8)$ \\
\hline Bone & $2(0.7)$ \\
\hline Other/unknown & $10(3.7)$ \\
\hline Sepsis, $n(\%)$ & $126(46)$ \\
\hline Septic shock, $n(\%)$ & $148(54)$ \\
\hline SOFA (points) & $10.6 \pm 5.1$ \\
\hline SAPS II (points) & $51.5 \pm 19.9$ \\
\hline APACHE II (points) & $27.3 \pm 7.8$ \\
\hline ICU LOS (days) & $6.8 \pm 9$ \\
\hline Hospital LOS (days) & $23.6 \pm 21.5$ \\
\hline ICU mortality, $n(\%)$ & $53(19.3)$ \\
\hline 28-day-mortality, $n(\%)$ & $76(27.7)$ \\
\hline
\end{tabular}

Data are given as mean values $\pm \mathrm{SD}$, if not indicated otherwise $c A H T$ chronic arterial hypertension, $C H D$ coronary heart disease, $C H F$ congestive heart failure, $C O P D$ chronic obstructive pulmonary disease, $C R I$ chronic renal insufficiency, $C L D$ chronic liver disease, CNS central nervous system, SOFA sequential organ failure assessment, SAPS simplified acute physiology score, APACHE acute physiologic and chronic health evaluation, $I C U$ intensive care unit, LOS length of stay

$11.9 \% ; P=0.006)$ and 28 -day-mortality (37.2 vs. $16.7 \%$; $P<0.001)$ than those without shock. Survivors had a higher MAP and MPP but a lower central venous pressure, mean pulmonary artery blood pressure, SOFA score, SAPS II, APACHE II, and longer hospital length of stay (Table 2). Moreover, they required catecholamines and renal replacement therapy less often than nonsurvivors.

Association of different ABP levels and 28-daymortality

There was a significant association between the hourly blood pressure time integral of SAP, MAP, as well as MPP and 28-day-mortality (Fig. 1). The areas under the ROC curve were largest for the hourly time integrals of ABP drops below MAP $60 \mathrm{mmHg}$ and MPP $45 \mathrm{mmHg}$.
No significant association between the hourly time integrals of ABP drops below certain SAP levels and 28-daymortality could be detected (Table 3 ). While the areas under the ROC curve were not different between the hourly time integrals of ABP drops below single MPP levels, the area under the ROC curve of the hourly time integral of ABP drops below MAP $60 \mathrm{mmHg}$ was higher than that of MAP $55(P=0.01), 50(P=0.002)$ and $45 \mathrm{mmHg} \quad(P<0.001)$. There were no differences between the areas under the ROC curves of the hourly time integrals of ABP drops below MAP $60 \mathrm{mmHg}$ and MAP levels $\geq 65 \mathrm{mmHg}$.

Patients with one or more hypotensive episodes (MAP $<60 \mathrm{mmHg}$ for $\geq 2 \mathrm{~min}$ ) had a higher 28-daymortality than patients without such a hypotensive episode during the observation period (29.8 vs. $12.5 \%$; OR, 2.96; CI 95\%, 1.06-10.36; $P=0.04$ ). There was a linear association between the time below MAP $60 \mathrm{mmHg}$ and 28-day-mortality (Fig. 2). Survivors had a shorter time below MAP $60 \mathrm{mmHg}$ than nonsurvivors (Table 4). When including only patients who required catecholamines into the binary logistic regression analysis, the areas under the ROC curve were similarly largest for the hourly time integrals of ABP drops below MAP 60 and MPP $45 \mathrm{mmHg}$.

Association with organ function (Table 5)

The hourly blood pressure time integrals of measured SAP, MAP and MPP were associated with maximum SOFA score, arterial lactate concentrations, need for renal replacement therapy, maximal serum creatinine concentrations, and hourly urine output, but not with liver function parameters. Inclusion of covariates (age, hourly cardiac index time integral, chronic arterial hypertension, catecholamines, analgosedative drugs) did not influence the association between $\mathrm{ABP}$ and renal function. The areas under the ROC curve to predict the need for renal replacement therapy were highest for the hourly time integrals of ABP drops below MAP $75 \mathrm{mmHg}$ (AUC ROC, 0.724; Sensitivity, 2.1\%; Specificity, 97.8\%; Positive Predictive Value, 16.7\%; Negative Predictive Value, 82.7\%; $P<0.001$ ) and MPP $60 \mathrm{mmHg}$ (AUC ROC, 0.721; Sensitivity, 11.4\%; Specificity, 97.8\%; Positive Predictive Value, 55.6\%; Negative Predictive Value, $82 \% ; P<0.001)$.

\section{Discussion}

In this study, we analyzed ABP in the first $24 \mathrm{~h}$ after ICU admission because of sepsis or septic shock since early cardiovascular failure is likely to influence subsequent organ function and outcome [22]. Because the first $24 \mathrm{~h}$ in 


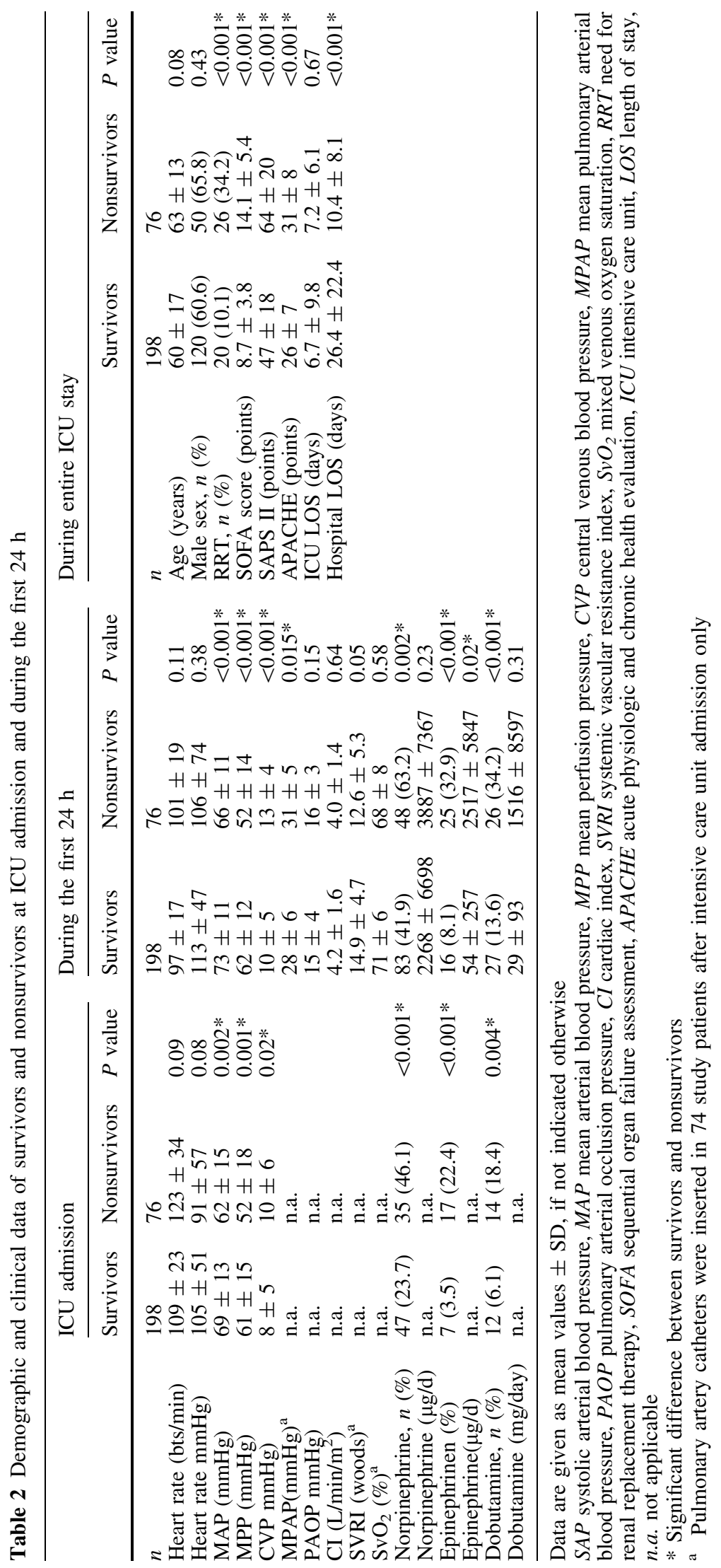



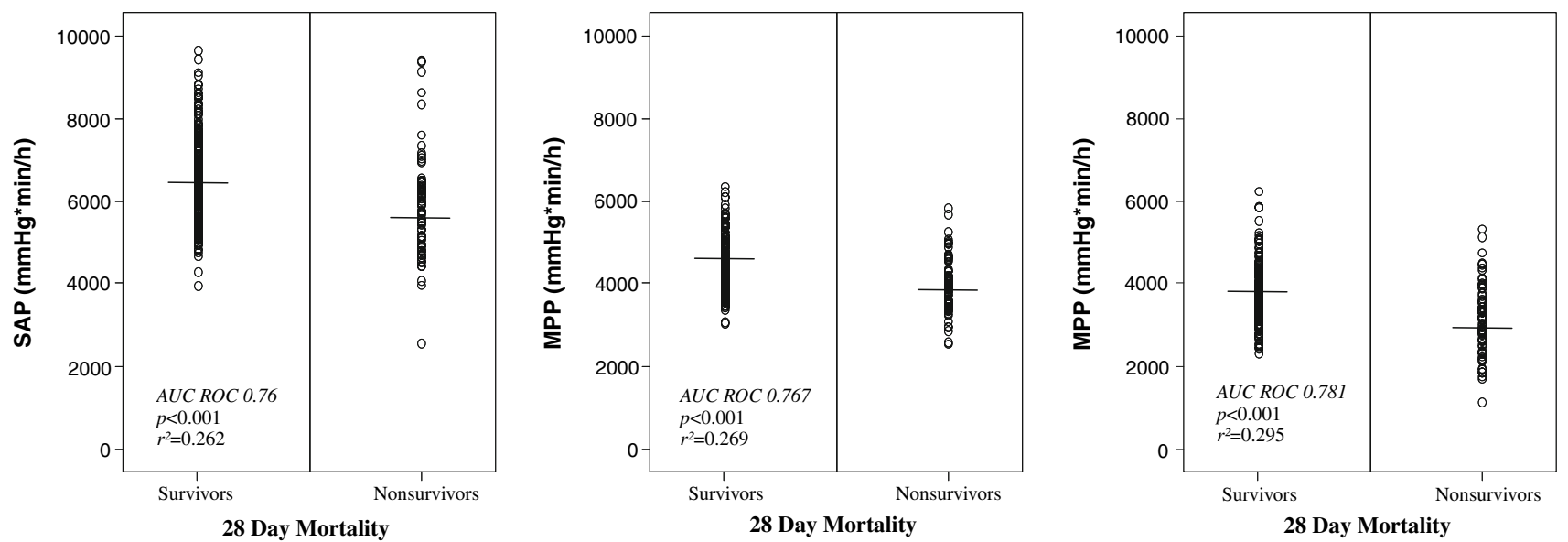

Fig. 1 Results are based on a binary logistic regression model curve of the hourly blood pressure time integral of the respective which was adjusted for disease severity as assessed by the arterial blood pressures. SAP hourly blood pressure time integral of Simplified Acute Physiology Score II (excl. SAP) during the first systolic arterial blood pressure, MAP hourly blood pressure time $24 \mathrm{~h}$ after intensive care unit admission. The AUC ROC (calculated integral of mean arterial blood pressure, MPP hourly blood from the results of the binary logistic regression analysis) pressure time integral of mean perfusion pressure represents the area under the receiver operating characteristic

Table 3 Association between different arterial blood pressure levels and 28-day-mortality adjusted for disease severity

\begin{tabular}{|c|c|c|c|c|c|c|c|c|}
\hline & $n^{\mathrm{a}}$ & Mean $\pm \mathrm{SD}^{\mathrm{b}}$ & AUC ROC & Sens $(\%)$ & Spec $(\%)$ & PPV $(\%)$ & NPV $(\%)$ & $P$ value \\
\hline \multicolumn{9}{|l|}{ SAP } \\
\hline HTI of ABP drops $<95 \mathrm{mmHg}$ SAP & 246 & $308 \pm 604$ & 0.743 & 93.4 & 29 & 77.4 & 62.9 & 0.06 \\
\hline HTI of ABP drops $<90 \mathrm{mmHg}$ SAP & 235 & $212 \pm 542$ & 0.737 & 94.4 & 26.3 & 77 & 64.5 & 0.12 \\
\hline HTI of ABP drops $<85 \mathrm{mmHg} \mathrm{SAP}$ & 217 & $143 \pm 489$ & 0.734 & 93.4 & 25 & 76 & 59.4 & 0.22 \\
\hline HTI of ABP drops $<80 \mathrm{mmHg}$ SAP & 189 & $96 \pm 444$ & 0.731 & 94.4 & 26.3 & 77 & 64.5 & 0.4 \\
\hline HTI of ABP drops $<75 \mathrm{mmHg}$ SAP & 159 & $67 \pm 406$ & 0.731 & 94.4 & 26.3 & 77 & 64.5 & 0.63 \\
\hline HTI of ABP drops $<70 \mathrm{mmHg}$ SAP & 124 & $50 \pm 373$ & 0.731 & 94.4 & 26.3 & 77 & 64.5 & 0.84 \\
\hline HTI of ABP drops $<65 \mathrm{mmHg}$ SAP & 77 & $38 \pm 343$ & 0.731 & 94.4 & 26.3 & 77 & 64.5 & 0.99 \\
\hline \multicolumn{9}{|l|}{ MAP } \\
\hline HTI of ABP drops $<75 \mathrm{mmHg}$ MAP & 261 & $475 \pm 388$ & 0.775 & 93.4 & 42.1 & 80.7 & 71.1 & $<0.001 * *$ \\
\hline HTI of ABP drops $<70 \mathrm{mmHg}$ MAP & 252 & $297 \pm 303$ & 0.777 & 94.9 & 40.8 & 80.6 & 75.6 & $<0.001 * *$ \\
\hline HTI of ABP drops $<65 \mathrm{mmHg}$ MAP & 245 & $162 \pm 217$ & 0.778 & 95.9 & 39.5 & 80.4 & 79 & $<0.001 * *$ \\
\hline HTI of ABP drops $<60 \mathrm{mmHg}$ MAP & 220 & $74 \pm 141$ & 0.779 & 95.4 & 39.5 & 80.3 & 76.9 & $<0.001 * *$ \\
\hline HTI of ABP drops $<55 \mathrm{mmHg}$ MAP & 177 & $30 \pm 86$ & 0.764 & 94.9 & 32.9 & 78.6 & 71.4 & $0.001 * *$ \\
\hline HTI of ABP drops $<50 \mathrm{mmHg}$ MAP & 135 & $11 \pm 49$ & 0.757 & 94.9 & 26.3 & 77 & 66.7 & 0.02 \\
\hline HTI of ABP drops $<45 \mathrm{mmHg}$ MAP & 85 & $5 \pm 28$ & 0.751 & 94.4 & 29 & 77.5 & 66.7 & 0.05 \\
\hline HTI of ABP drops $<$ targeted MAP & 159 & $56 \pm 113$ & 0.769 & 95.4 & 37.3 & 78.1 & 80 & $0.001 *$ \\
\hline \multicolumn{9}{|l|}{ MPP } \\
\hline HTI of ABP drops $<60 \mathrm{mmHg}$ MPP & 210 & $398 \pm 432$ & 0.788 & 92.4 & 42.9 & 74.4 & 71.4 & $<0.001 * *$ \\
\hline HTI of ABP drops $<55 \mathrm{mmHg}$ MPP & 200 & $252 \pm 343$ & 0.791 & 92.4 & 41.4 & 78 & 70.7 & $<0.001 * *$ \\
\hline HTI of ABP drops $<50 \mathrm{mmHg}$ MPP & 177 & $147 \pm 254$ & 0.793 & 92.4 & 40 & 77.5 & 70 & $<0.001 * *$ \\
\hline HTI of ABP drops $<45 \mathrm{mmHg}$ MPP & 159 & $77 \pm 175$ & 0.797 & 92.4 & 40 & 77.5 & 70 & $<0.001 * *$ \\
\hline HTI of ABP drops $<40 \mathrm{mmHg}$ MPP & 128 & $38 \pm 111$ & 0.793 & 93 & 38.6 & 77.3 & 71.1 & $0.001 * *$ \\
\hline HTI of ABP drops $<35 \mathrm{mmHg}$ MPP & 90 & $17 \pm 63$ & 0.792 & 93.6 & 38.6 & 77.4 & 73 & 0.01 \\
\hline HTI of ABP drops $<30 \mathrm{mmHg}$ MPP & 60 & $7 \pm 31$ & 0.782 & 90.5 & 37.1 & 76.3 & 63.4 & 0.02 \\
\hline
\end{tabular}

The hourly time integral (HTI) of arterial blood pressure (ABP) drops below certain pressure limits represents the duration and extent of blood pressure drops below the respective arterial blood pressure level per hour. Results are based on a binary logistic regression model which was adjusted for disease severity as assesed by the Simplified Acute Physiology Score II count (excl. SAP) during the first $24 \mathrm{~h}$ after intensive care unit admission. The targeted MAP was available in 240 study patients

$S A P$ systolic arterial blood pressure, $A U C R O C$ area under the receiver operating characteristic curve, Sens sensitivity, Spec specifity, $P P V$ positive predictive value, $N P V$ negative predictive value, $M A P$ mean arterial blood pressure, $M P P$ mean perfusion pressure

** Significant association between ABP target and mortality

a Number of patients who experienced an arterial blood pressure drop below the given limit

${ }^{b}$ Mean values \pm SD refer to the HTI of ABP drops below certain arterial blood pressure levels and are given in $\mathrm{mmHg} \mathrm{min} / \mathrm{h}$ 


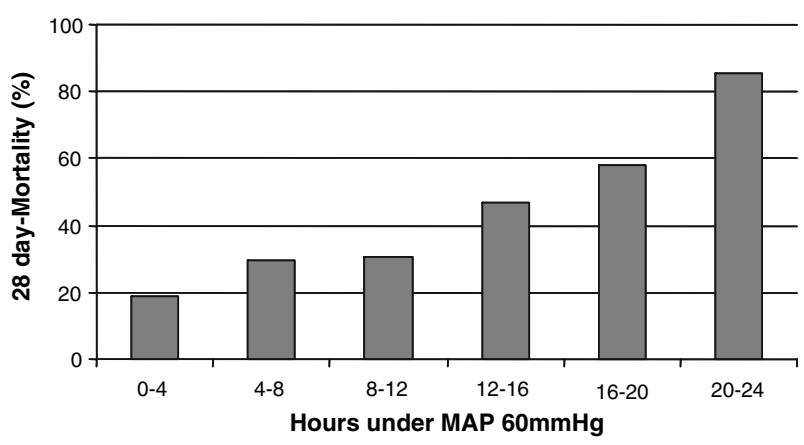

Fig. 2 The histogram shows the association between 28-daymortality and the total time spent below a MAP level of $60 \mathrm{mmHg}$ during the first $24 \mathrm{~h}$ after intensive care unit admission in all study patients. The number of patients in each time range was $0-4 \mathrm{~h}$, $n=180 ; 4-8$ h, $n=37 ; 8-12 \mathrm{~h}, n=20 ; 12-16 \mathrm{~h}, n=20 ; 16-20 \mathrm{~h}$, $n=10$; and $20-24 \mathrm{~h}, n=7$. MAP mean arterial blood pressure. * Significant difference between survivors and nonsurvivors

the ICU do not necessarily represent the start of the disease process, the lead-time bias is difficult to quantify and contributes to the presence and successive development of hemodynamic and remote organ dysfunction. Nevertheless, the association between ABP during the early phase of sepsis and septic shock and outcome in the present study has also been reported by others [2, 3, 13, 23]. In this study, ABP was associated with 28-day-mortality even when corrected for the impact of disease severity.

Currently, a MAP of $65-90 \mathrm{mmHg}$ is a widely accepted and recommended blood pressure range for critically ill sepsis patients $[7-9,24]$. In our study population, there was no difference in the risk of death at 28 days between ABP drops below MAP of $60,65,70$ and $75 \mathrm{mmHg}$. When analysing the hourly time integral of ABP drops below a MAP of $55 \mathrm{mmHg}$, a significant decrease in the area under the ROC curve was observed. This suggests that a MAP of $60 \mathrm{mmHg}$ during the first $24 \mathrm{~h}$ of ICU therapy represents a critical level in our study population. Since the ABP target determines the definition of septic shock, we chose to avoid this confounding factor and included all sepsis patients. Had only septic shock patients (e.g. patients with vasopressors, according to the targets set by the clinician) been included, critical ABP levels may have been different.

Furthermore, it needs to be considered that we analyzed the hourly time integral of $\mathrm{ABP}$ drops below certain
ABP levels. This method pools hourly ABP data but does not account for temporary variations and hence cannot be considered as the total amount of hypotension exposure over $24 \mathrm{~h}$. Despite of including 274 patients into this analysis, the statistical power may still be too low to reliably exclude a mortality difference between a MAP of $60 \mathrm{mmHg}$ and higher MAP levels. Since our analysis primarily evaluated ABP levels and no specific treatment goals, we can neither determine whether the critical MAP level of $60 \mathrm{mmHg}$ may equally represent the optimum therapeutic goal. In order to evaluate the influence of a MAP goal of $\geq 60 \mathrm{mmHg}$ on mortality in sepsis patients, future prospective trials are needed. Such studies could also provide information if a safety margin (e.g. $5 \mathrm{mmHg}$ ) above the critical MAP level of $60 \mathrm{mmHg}$ may be useful to avoid detrimental hypotensive episodes or would increase catecholamine requirements without exerting beneficial effects on tissue perfusion and mortality.

In a recent study, Varpula et al. [13], using ROC analysis, identified a MAP of $65 \mathrm{mmHg}$ as the best limit to discriminate surviving and nonsurviving septic shock patients. This difference to our study may be explained by the lack of correction for disease severity. Whereas the APACHE II Score count was higher in our than in Varpula's patient population, the degree of multiple organ dysfunction appears comparable when considering SOFA score counts of the two study groups. When a simple, unadjusted ROC analysis is applied to our data, a MAP of $65 \mathrm{mmHg}$ similarly shows the largest area under the ROC curve for 28-day-mortality (Electronic Repository). MAP was also the hemodynamic parameter which was most significantly associated with 28-day-mortality in a multiple logistic regression model (Electronic Repository).

MPP also showed a significant association with mortality. According to a well-established physiologic, but clinically rarely applied concept, organ blood flow is determined by the pressure gradient (arterial-venous pressure) across the organ vasculature divided by the total resistance of the organ vascular bed [25]. Despite the physiologic limitations of central venous pressure to reflect organ specific venous pressure, MPP includes an estimation of perfusion pressure. It is therefore conceivable that it should correlate equally well or better with mortality and renal function than SAP or MAP alone. However, the current value of MPP as a therapeutic target is unproven and needs to be addressed in clinical trials.

Table 4 Hourly and daily time in minutes spent below a mean arterial blood pressure of $60 \mathrm{mmHg}$ in survivors and nonsurvivors

\begin{tabular}{|c|c|c|c|c|c|}
\hline & \multicolumn{2}{|l|}{ Survivors } & \multicolumn{3}{|l|}{ Nonsurvivors } \\
\hline & Mean \pm SD & CI $95 \%$ & Mean \pm SD & CI $95 \%$ & $P$ value \\
\hline $\mathrm{MAP}<60 \mathrm{mmHg} / \mathrm{h}(\mathrm{min})$ & $9 \pm 13$ & $7-11$ & $22 \pm 20$ & $17-26$ & $<0.001 *$ \\
\hline MAP $<60 \mathrm{mmHg} /$ day (min) & $195 \pm 272$ & $157-233$ & $455 \pm 433$ & $356-554$ & $<0.001 *$ \\
\hline
\end{tabular}

$M A P$ mean arterial blood pressure 
Table 5 Association between the hourly blood pressure time integrals of measured arterial blood pressures and organ functions as well as the maximum SOFA score

\begin{tabular}{llllll}
\hline & \multicolumn{2}{l}{ SOFA } & & \multicolumn{2}{c}{ Max. lactate } \\
\cline { 2 - 3 }$n=190$ & $r^{2}$ & $P$ value & & $r^{2}$ & $P$ value \\
\hline HBPTI of SAP & 0.267 & $0.009^{*}$ & & 0.210 & $<0.001^{*}$ \\
HBPTI of MAP & 0.268 & $0.012^{*}$ & & 0.196 & $<0.001^{*}$ \\
HBPTI of MPP & 0.233 & $0.013^{*}$ & & 0.207 & $<0.001^{*}$ \\
\hline
\end{tabular}

Liver function Liver failure Max. ASAT Max. tBilirubin

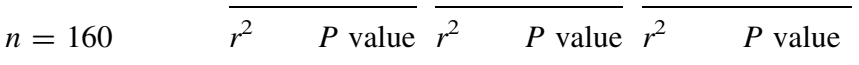

$\begin{array}{lllllll}\text { HBPTI of SAP } & 0.077 & 0.394 & 0.053 & 0.721 & 0.012 & 0.881\end{array}$

$\begin{array}{lllllll}\text { HBPTI of MAP } & 0.083 & 0.336 & 0.054 & 0.738 & 0.022 & 0.307\end{array}$

$\begin{array}{lllllll}\text { HBPTI of MPP } & 0.072 & 0.702 & 0.045 & 0.841 & 0.027 & 0.595\end{array}$

\begin{tabular}{|c|c|c|c|c|c|c|}
\hline \multirow{2}{*}{$\begin{array}{l}\text { Renal function* } \\
n=274\end{array}$} & \multicolumn{2}{|l|}{ RRT } & \multicolumn{2}{|c|}{ Max. Creatinine } & \multicolumn{2}{|c|}{ Urine output/h } \\
\hline & $r^{2}$ & & $r^{2}$ & & $r^{2}$ & \\
\hline & & & & & & \\
\hline & 0. & & & & & $01 *$ \\
\hline HBPTI of & 0.136 & $<0.001^{*}$ & 0.277 & $0.002 *$ & 0.273 & $<0.001^{*}$ \\
\hline
\end{tabular}

The hourly blood pressure time integral (HBPTI) represents the hourly area under the measured arterial blood pressures. Results are based on a binary logistic regression model which was adjusted for disease severity as assesed by the Simplified Acute Physiology Score II (excl. SAP) during the first $24 \mathrm{~h}$ after ICU admission SOFA sequential organ failure assessment; max., maximum, SAP systolic arterial blood pressure, MAP mean arterial blood pressure, $M P P$ mean perfusion pressure, ASAT aspartate aminotransferase, tBilirubin total bilirubin, $R R T$ need for renal replacement therapy * Significant association between arterial blood pressure and organ function or arterial lactate levels

The observation that ABP during the first $24 \mathrm{~h}$ was associated with SOFA score and arterial lactate concentrations supports and may partly explain the association between $\mathrm{ABP}$ and mortality. However, given the infusion of epinephrine in $\sim 15 \%$ of patients, arterial lactate levels may not have reflected tissue hypoperfusion in all patients [26]. Together with the fact that several further therapeutic steps influence 28 -day-mortality in sepsis patients, this may explain why only low $r^{2}$ values were detected for the association between ABP and organ functions. Out of all organs, the kidney is known to have the highest autoregulation threshold [25, 27, 28]. Accordingly, the MAP and MPP levels to discriminate study patients who did or did not require renal replacement therapy were 75 and $60 \mathrm{mmHg}$, respectively. Since these were the highest MAP and MPP levels tested, it cannot be excluded that the critical ABP levels for renal function are even higher. While a small-size prospective study found no influence of increasing MAP from 65 to $85 \mathrm{mmHg}$ with norepinephrine on renal function in septic shock patients [12], Deruddre et al. [29] suggested that Doppler ultrasonography and resistive index measurements could help determine the optimum MAP for renal blood flow in septic shock.

When interpreting the results of this study important limitations need to be considered. A major limitation is the retrospective and uncontrolled design. Since a guideline instead of a strict protocol was used, some variations in therapeutic practice were certainly present. Despite automated capture of frequently sampled filtered data, missing data due to interventions and diagnostic procedures cannot be avoided. Considering the amount of data available for analysis and the use of mean values over $24 \mathrm{~h}$, this is likely to have only a minor influence on the association between ABP and mortality. Because only patients monitored with an arterial line were included, a selection bias by excluding less severe sepsis patients who could be managed with non-invasive ABP measurements may have occurred.

In conclusion, a MAP level $\geq 60 \mathrm{mmHg}$ may be as safe as higher MAP levels during the first $24 \mathrm{~h}$ of ICU therapy in septic patients. A higher MAP may be required to maintain kidney function. Future randomized clinical trials are necessary to evaluate the MAP targets in sepsis.

Acknowledgments The authors are indebted to Mrs. Pia Burri and Mr. Roy Lanz for their invaluable assistance in extracting the study variables from the database.

\section{References}

1. Mayr VD, Dünser MW, Greil V, Jochberger S, Luckner G, Ulmer H, Friesenecker BE, Takala J, Hasibeder WR (2006) Causes of death and determinants of outcome in critically ill patients. Crit Care 10:R154

2. Kumar A, Roberts D, Wood KE, Light B, Parrillo JE, Sharma S, Suppes R, Feinstein D, Zanotti S, Taiberg L, Gurka D, Kumar A, Cheang M (2006) Duration of hypotension before initiation of effective antimicrobial therapy is the critical determinant of survival in human septic shock. Crit Care Med 34:1589-1596
3. Bernardin G, Pradier C, Tiger F, Deloffre P, Mattei M (1996) Blood pressure and arterial lactate level are early indicators of short-term survival in human septic shock. Intensive Care Med 22:17-25

4. Martin C, Viviand X, Leone M, Thirion $X$ (2000) Effect of norepinephrine on the outcome of septic shock. Crit Care Med 28:2758-2765
5. Dünser MW, Mayr AJ, Ulmer H, Knotzer H, Sumann G, Pajk W, Friesenecker B, Hasibeder WR' (2003) Arginine vasopressin in advanced vasodilatory shock: a prospective, randomized, controlled study. Circulation 107:2313-2319

6. Dellinger RP (2003) Cardiovascular management of septic shock. Crit Care Med 31:946-955

7. Annane D, Bellissant E, Cavaillon JM (2005) Septic shock. Lancet 365:63-78 
8. Dellinger RP, Carlet JM, Masur H, Gerlach H, Calandra T, Cohen J, GeaBanacloche J, Keh D, Marshall JC, Parker MM, Ramsay G, Zimmerman JL, Vincent JL, Levy MM, Surviving Sepsis Campaign Management Guidelines Committee (2004) Surviving Sepsis Campaign guidelines for management of severe sepsis and septic shock. Crit Care Med 32:858873

9. Meier-Hellmann A, Reinhart K, Bredle DL, Specht M, Spies CD, Hannemann L (1997) Epinephrine impairs splanchnic perfusion in septic shock. Crit Care Med 25:399-404

10. Lopez A, Lorente JA, Steingrub J, Bakker J, McLuckie A, Willatts S, Brockway M, Anzueto A, Holzapfel L, Breen D, Silverman MS, Takala J, Donaldson J, Arneson C, Grove G, Grossman S, Grover R (2004) Multiplecenter, randomized, placebo-controlled, double-blind study of the nitric oxide synthase inhibitor 546C88: effect on survival in patients with septic shock. Crit Care Med 32:21-30

11. LeDoux D, Astiz ME, Carpati CM, Rackow EC (2000) Effects of perfusion pressure on tissue perfusion in septic shock. Crit Care Med 28:2729-2732

12. Bourgoin A, Leone M, Delmas A, Garnier F, Albanese J, Martin C (2005) Increasing mean arterial pressure in patients with septic shock: effects on oxygen variables and renal function. Crit Care Med 33:780-786

13. Varpula M, Tallgren M, Saukkonen K, Voipio-Pulkki LM, Pettilä V (2005) Hemodynamic variables related to outcome in septic shock. Intensive Care Med 31:1066-1071

14. Dünser MW, Daudel F, Luckner G, Mayr V, Jochberger S, Hasibeder WR, Takala J, Jakob S (2007) Arterial blood pressure during early sepsis. Intensive Care Med 33(Suppl 2):A125

15. Levy MM, Fink MP, Marshall JC, Abraham E, Angus D, Cook D, Cohen J, Opal SM, Vincent JL, Ramsay G, SCCM/ESICM/ACCP/ATS/SIS (2003) 2001 SCCM/ESICM/ACCP/ATS/SIS International Sepsis Definitions Conference. Crit Care Med 31:12501256
16. Makivirta A, Koski E, Kari A, Sukuvaara T (1991) The median filter as a preprocessor for a patient monitor limit alarm system in intensive care. Comput Methods Programs Biomed 34:139-144

17. Le Gall JR, Lemeshow S, Saulnier F (1993) A new Simplified Acute Physiology Score (SAPS II) based on a European/North American multicenter study. JAMA 270:2957-2963

18. Knaus WA, Zimmerman JE, Wagner DP, Draper EA, Lawrence DE (1981) APACHE - acute physiology and chronic health evaluation: a physiologically based classification system. Crit Care Med 9:591-597

19. Jakob SM, Korhonen I, Ruokonen E, Virtanen T, Kogan A, Takala J (2000) Detection of artifacts in monitored trends in intensive care. Comput Methods Programs Biomed 63:203-209

20. Vincent JL, Moreno R, Takala J, Willatts S, De Mendonca A, Bruining H, Reinhart CK, Suter PM, Thijs LG (1996) The SOFA (Sepsis-related Organ Failure Assessment) score to describe organ dysfunction/failure. On behalf of the working group on sepsisrelated problems of the european society of intensive care medicine. Intensive Care Med 22:707-710

21. DeLong ER, DeLong DM, ClarkePearson DL (1988) Comparing the areas under two or more correlated receiver operating curves: a nonparametric approach. Biometrics 44:837-845

22. Rivers E, Nguyen B, Havstad S, Ressler J, Muzzin A, Knoblich B, Peterson E, Tomlanovich M, Early Goal-Directed Therapy Collaborative Group (2001) Early goal-directed therapy in the treatment of severe sepsis and septic shock. N Engl J Med 345:1368-1377
23. Jones AE, Yiannibas V, Johnson C, Kline JA (2006) Emergency department hypotension predicts sudden unexpected in-hospital mortality. A prospective cohort study. Chest 130:941-946

24. Beale RJ, Hollenberg SM, Vincent JL, Parrillo JE (2004) Vasopressor and inotropic support in septic shock: an evidence-based review. Crit Care Med 32:S455-465

25. Guyton AC, Hall JE (2000) Urine formation by the kidneys: I. Glomerular filtration, renal blood flow, and their control. In: Guyton AC, Hall JE (eds) Textbook of medical physiology. Saunders, Philadelphia, pp 279-294

26. Levy B, Gibot S, Franck P, Cravoisy A, Bollaert PE (2005) Relation between muscle $\mathrm{Na}+\mathrm{K}+$ ATPase activity and raised lactate concentrations in septic shock: a prospective study. Lancet 365:871-875

27. Rowell LB (1986) Control of individual vascular beds: splanchnic and renal circulations. In: Rowell LB (ed) Human circulation. Regulation during physical stress. Oxford University Press, Oxford, pp 78-95

28. Bellomo R, Kellum JA, Wisniewski SR, Pinsky MR (1999) Effects of norepinephrine on the renal vasculature in normal and endotoxemic dogs. Am J Respir Crit Care Med 159:1186-1192

29. Deruddre S, Cheisson G, Mazoit JX, Vicaut E, Benhamou D, Duranteau J (2007) Renal arterial resistance in septic shock: effects of increasing mean arterial pressure with norepinephrine on the renal resistive index with Doppler ultrasonography. Intensive Care Med 33:1557-1562 